\title{
Feasibility and efficacy of shared decision making for first-admission schizophrenia: a randomized clinical trial
}

\author{
Mio Ishii ${ }^{1}$, Yasuyuki Okumura2* (D), Naoya Sugiyama ${ }^{3}$, Hana Hasegawa $^{3}$, Toshie Noda ${ }^{3}$, Yoshio Hirayasu ${ }^{1}$ \\ and Hiroto Ito $^{4}$
}

\begin{abstract}
Background: The feasibility of shared decision making (SDM) for patients with schizophrenia remains controversial due to the assumed inability of patients to cooperate in treatment decision making. This study evaluated the feasibility and efficacy of SDM in patients upon first admission for schizophrenia.

Methods: This was a randomized, parallel-group, two-arm, open-label, single-center study conducted in an acute psychiatric ward of Numazu Chuo Hospital, Japan. Patients with the diagnosis of schizophrenia upon their first admission were randomized into a SDM intervention group or a usual treatment group in a 1:1 ratio. The primary outcome was patient satisfaction at discharge. The secondary outcomes were attitudes toward medication at discharge and treatment continuation at 6 months after discharge.

Results: Twenty-four patients were randomly assigned. The trial was prematurely terminated due to slow enrollment. At discharge, the mean score on satisfaction was 23.7 in the SDM group and 22.1 in the usual care group (unadjusted mean difference: $1.6 ; 95 \% \mathrm{Cl}$ : -5.2 to 2.0). Group differences were not observed in attitude toward medication and treatment continuation. There was no statistically significant difference between the groups for the mean Global Assessment of Functioning score at discharge or length of stay as safety endpoint.

Conclusions: No statistical differences were found between the SDM group and usual care group in the efficacy outcomes and safety endpoints. Large trials are needed to confirm the efficacy of the SDM program upon first admission for schizophrenia.
\end{abstract}

Trial registration: The study has been registered with ClinicalTrials.gov as NCT01869660 (registered 27 May, 2013).

Keywords: Shared decision making, SDM, Schizophrenia, Randomized controlled trial, Pilot study, Inpatient treatment

\section{Background}

Patient-centered care, where patients and health service providers establish partnerships to ensure every decision for patients' needs and preferences, has been a core component of modern general medicine [1, 2]. Shared decision making (SDM) is a model in which patients and clinicians collaborate together throughout the entire decision-making process, with information shared and patients allowed to state their individual preferences [3].

\footnotetext{
* Correspondence: yokumura@blue.zero.jp

${ }^{2}$ Research Department, Institute for Health Economics and Policy, Association for Health Economics Research and Social Insurance and Welfare, 1-5-11 Nishishimbashi, Minato-ku, Tokyo 105-0003, Japan

Full list of author information is available at the end of the article
}

SDM is expected to embody the idea of patient-centered care in clinical medicine. A meta-analysis found that, compared with usual care, SDM improves a patients' knowledge acquisition, confidence, and active participation in treatment [4].

On the other hand, there has been some controversy among psychiatrists regarding the feasibility of SDM in regards to psychiatric treatment, particularly when it involves the treatment of severe mental illnesses such as schizophrenia, with respect to patient vulnerability to paternalism and coercive treatment due to patient symptoms such as disorganized thinking and excessive suspicion $[5,6]$. Therefore, SDM had not targeted patients with severe mental illness such as schizophrenia because 
of presumed inability to cooperate in treatment decision making. Along with the recovery movement, mental healthcare providers are now encouraged to support every patient in pursuing their life goals [7]. In addition, clinical guidelines $[8,9]$ now advocate the use of SDM in psychiatry as a patient-centered care.

Nevertheless, there is a lack of evidence of SDM in patients with severe mental illness. To the best of our knowledge, only five randomized controlled trials have assessed the efficacy of SDM in patients with schizophrenia and related disorders [10-14]. Furthermore, there are serious limitations with the previous studies. First, only two trials $[10,11]$ focused on inpatient treatments in which coercive treatments are commonly administered. Second, all trials, with the exception of one that assessed long-term adherence and readmission rates [11], used only soft outcomes such as treatment satisfaction, perceived involvement in treatment decision making, and participants' feelings of empowerment. Third, no study focused a homogenous sample of patients who do not have a history of psychiatric hospitalization. We believe it is important to focus on patients without prior experience of hospitalization, because better relationships between patients and medical providers in the early treatment phase for schizophrenia lead to better compliance [15].

Additional trials are needed to establish the feasibility and efficacy of SDM in patients with schizophrenia. We aimed to evaluate the feasibility and efficacy of SDM in patients upon first admission for schizophrenia.

\section{Methods}

\section{Design overview}

Details of the study protocol have been reported elsewhere [16]. The objective of this study was to evaluate the feasibility and efficacy of an SDM intervention compared to usual treatment on patient satisfaction at discharge and treatment continuation 6 months post-discharge for firstadmission patients with schizophrenia. This was an investigator-initiated, single-center, randomized trial in which participants were randomly assigned either SDM plus usual care or usual care. Assessments were completed at admission, discharge, and 6 months after discharge. The trial was registered at ClinicalTrials.gov (NCT01869660) on May 27, 2013, and the first patient was recruited on June 4, 2013, and the last patient completed follow-up on September 29, 2015. The present study was originally planned to recruit 58 patients but was stopped prematurely due to slow patient enrollment. The institutional review board of the Yokohama City University, Japan, approved this study (No.: A130321008). All participants provided written informed consent or, if the participant was younger than 20 years of age, the legal guardian provided written informed consent.

\section{Setting and participants}

Participants were consecutively recruited from an acute psychiatric ward of the Numazu Chuo Hospital, located in Numazu city, Shizuoka, Japan. Numazu Chuo Hospital is the only psychiatric hospital that receives emergency admissions for a population of 870,115 inhabitants. We assessed the following eligibility criteria within 3 days of admission: patients were aged 16-65 years; had no history of psychiatric admission (first admission); had a diagnosis of schizophrenia spectrum disorder (including schizophrenia, schizotypal, and delusional disorders defined according to the diagnosis codes F20-F29 in the International Statistical Classification of Diseases, 10th revision [ICD-10]). Exclusion criteria included moderate to profound mental retardation, organic mental disorders (ICD-10 codes: F00-F09), inability to converse in Japanese, and severe conceptual disorganization (a score of 5 or more on the Brief Psychiatric Rating Scale) [17, 18].

\section{Randomization}

Randomization was conducted by the central web-based randomization system after obtaining written informed consent. Participants were randomly assigned in a 1:1 ratio using a computer-generated random number sequence. Assignment was balanced for the stratification factors of sex, age (younger or older than 20 years of age), and onset of illness (less or more than 1 year) by using a minimization method.

\section{Interventions}

In the SDM group, participants received the SDM model program in addition to usual psychiatric inpatient care. The SDM model program is a 15-20-min weekly intervention during the acute psychiatric ward stay, and its development has been detailed previously [16]. The intervention consists of three sequential elements: assessing patient's perceptions on their on-going treatments by a self-report questionnaire; sharing patients' and medical staffs' perceptions on the treatments in a 15-20-min meeting; and patients together with medical staff deciding on a care plan for the next week. As a medical team, a primary physician, a primary nurse, and others participated in the meetings. To improve adherence and quality of the intervention, independent supervisors managed intervention schedules, facilitated meetings, and educated medical staff.

In the usual care group, participants received usual psychiatric inpatient care, which is mainly pharmacological treatment. During the hospitalization, primary physicians examined their patients every day and nurses helped patients by focusing on self-care activities and the daily activity program. Primary physicians and nurses usually discussed the patient's overall progress and plan 
for discharge. However, there was no fixed occasion for the patient and staff to share all the information. In addition, there was no certainty that patients would be introduced to the concept of SDM or that they would be empowered to actively participate in the treatment.

Patients in both SDM and usual care groups received treatments by the same medical staff in the ward- 15 primary psychiatrists, nurses and three psychiatric social workers.

\section{Outcomes and follow-up}

The primary outcome was patient satisfaction at discharge. Patient satisfaction was assessed using the Japanese version of the Client Satisfaction Questionnaire (CSQ-8 J), a 8 -item self-rated measure $[19,20]$. The CSQ-8 J assesses overall satisfaction with care received using a four-point Likert scale. Scores range from 8 to 32 , with higher values indicating greater satisfaction. The CSQ-8 J has good internal consistency (Cronbach's $\alpha=.83)$ and moderate convergent validity $(r=.36-.49)$ with the client satisfaction inquiry [21].

Secondary outcomes included attitudes toward medication at discharge and treatment continuation 6 months after discharge. Attitudes were assessed using the Japanese version of the Drug Attitude Inventory-10 (DAI-10), a 10item self-rated measure $[22,23]$. The DAI-10 assess attitude toward medication using a true-false scale. Scores range from -10 to 10 , with higher values indicating more positive attitudes. The DAI-10 has good internal consistency (Cronbach's $\alpha=.97$ ) and adequate test-retest reliability $(r=.81)$ [23]. Treatment continuation was assessed by identifying whether a patient received outpatient psychiatric treatment within 30 days prior to the follow-up time (i.e., at 6 months after discharge). We identified information on treatment continuation from medical records if the patient continued treatment at the Numazu Chuo Hospital or if not, by asking patients with a telephone call. Symptom severity, as assessed by the Brief Psychiatric Rating Scales (BPRS) at discharge, was removed as a secondary outcome as this outcome was incorrectly specified in the original study protocol (14). We also amended the protocol to include the Global Assessment of Functioning at discharge [24] and length of stay as safety endpoints.

\section{Statistical analysis}

We estimated unadjusted mean difference (MD) and risk difference (RD) with their 95\% confidence interval (95\% $\mathrm{CI}$ ) between the groups (SDM vs. usual care) using Student's $t$ test for continuous variables (i.e., patient satisfaction and attitude toward medication) and chisquared test for a categorical variable (i.e., treatment continuation). We also calculated standardized MD and risk ratio with their $95 \%$ confidence interval between the groups. To control characteristics such as sex, age, duration of illness, and symptom severity assessed by the BPRS at admission, we estimated adjusted MD and RD using a marginal structural binomial model [25]. All data were analyzed by $\mathrm{R}$ version 3.2.0.

\section{Results}

\section{Baseline characteristics}

A total of 448 patients were screened, of whom 24 were randomly assigned (Fig. 1). The most common reason for ineligibility was a prior history of psychiatric admission $(n=$ 280). After randomization, two patients were lost before outcome assessments due to hospital transportation and early discharge without doctors' permission. Table 1 shows baseline characteristics in the groups. In general (87\% of total of 30 sessions), a primary doctor, a ward nurse, and a facilitator participated in the SDM meeting. The median number of sessions received was 3 (range: $2-5$ ).

\section{Outcomes}

For the primary and secondary outcomes, group differences were not observed in both unadjusted and adjusted analyses (Table 2). At discharge, the mean score on satisfaction was 23.7 in the SDM and 22.1 in usual care group (unadjusted MD: 1.6; 95\% CI: -5.2 to 2.0 ). The mean score of attitudes toward medication was 3.8 in the SDM and 2.3 in usual care group (unadjusted MD: 1.5 ; $95 \%$ CI: -5.6 to 2.7 ). At 6 months after discharge, continuation rate was $89 \%$ in the SDM group and $69 \%$ in usual care group (unadjusted RD: 19.7; 95\% CI: -12.8 to 52.1 ). There were no statistically significant differences between the groups for the mean Global Assessment of Functioning score at discharge or length of stay (Table 2).

\section{Discussion}

We examined whether our SDM intervention-a complex intervention including assessment of patient's perception, regular meetings, and shared care planning-improved clinical outcomes for patients with schizophrenia in the early treatment stage. This pilot study is the first randomized controlled trial to evaluate efficacy of SDM targeting patients with first-admitted schizophrenia. We found no statistical differences between the SDM group and usual care group in the primary and secondary outcomes.

Until now, SDM for patients with acute psychosis was deemed difficult because of the patient's decisional capacity, time constraints, and coercive atmosphere [26]. Consequently, medical providers have been hesitant about patient's active involvement in decision making. However, in our study, treatment adherence was maintained throughout the trial. All cases in the intervention group could receive interventional SDM sessions, for 20 min per week, for multiple times in spite of acute 


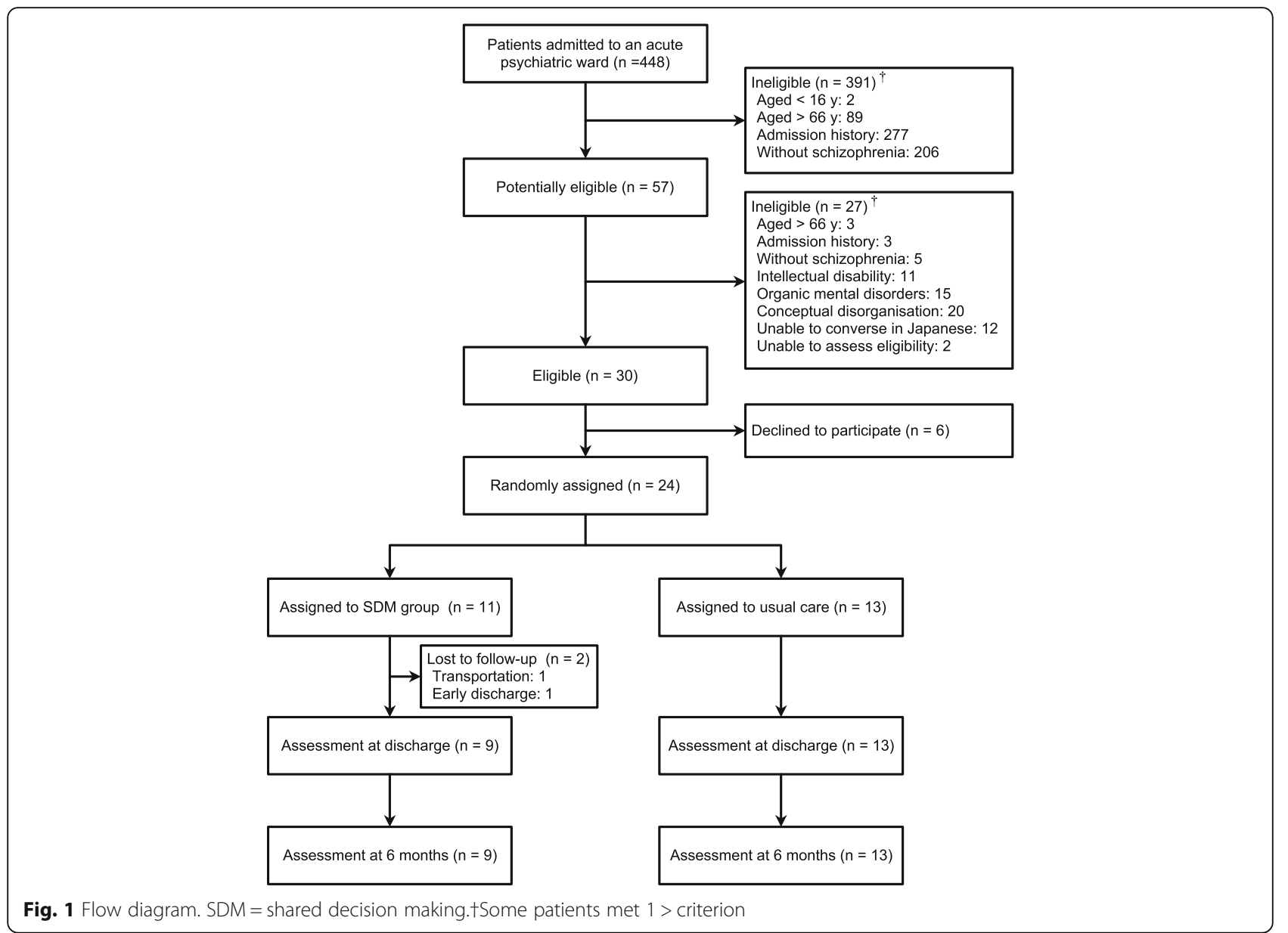

symptoms and busyness at the emergency ward. There were no statistically significant differences between groups in safety endpoints. None of the participants dropped out from the SDM intervention, with the exception of hospital transportation and early discharge without a doctor's permission. These results suggest the feasibility of SDM in patients with first-admission schizophrenia.

The strengths of our trial include a high rate of informed consent acquisition, assessment of treatment continuation after discharge as a hard endpoint, narrow eligibility criteria of first-admission schizophrenia, and

Table 1 Baseline characteristics

\begin{tabular}{llll}
\hline Characteristic & Total $(n=22)$ & SDM $(n=9)$ & Usual care $(n=13)$ \\
\hline Women, $n$ (\%) & $7(31.8)$ & $3(33.3)$ & $4(30.8)$ \\
Mean age at admission (SD), y & $39.1(11.7)$ & $41.6(13.6)$ & $37.4(9.8)$ \\
At least 1-year after the onset of illness, $n(\%)$ & $14(63.6)$ & $7(77.8)$ & $7(53.8)$ \\
Suicidal ideation, $n$ (\%) & & & $5(38.5)$ \\
$\quad$ Absent & $8(36.4)$ & $3(33.3)$ & $4(30.8)$ \\
$\quad$ Present & $9(40.9)$ & $5(55.6)$ & $4(30.8)$ \\
$\quad$ Unknown & $5(22.7)$ & $1(11.1)$ & $2(15.4)$ \\
Physical comorbidity, $n$ (\%) & $6(27.3)$ & $4(44.4)$ & $62.2(12.9)$ \\
Mean BPRS score at admission (SD) & $57.0(14.8)$ & $49.6(14.2)$ & $14.2(6.4)$ \\
Mean GAF score at admission (SD) & $17.5(12.5)$ & $66.9(17.1)$ & $65.2(50.5)$
\end{tabular}

BPRS the Brief Psychiatric Rating Scale, GAF the Global Assessment of Functioning scale, SDM shared decision making 
Table 2 Primary and secondary outcomes

\begin{tabular}{|c|c|c|c|c|c|}
\hline Outcome & SDM & Usual care & $\begin{array}{l}\text { Crude treatment } \\
\text { effect }(95 \% \mathrm{Cl})\end{array}$ & $\begin{array}{l}\text { Adjusted treatment } \\
\text { effect }(95 \% \mathrm{CI})^{\mathrm{c}}\end{array}$ & $\begin{array}{l}\text { Standardized mean } \\
\text { difference/risk ratio (95\% Cl) }\end{array}$ \\
\hline \multicolumn{6}{|l|}{ Primary outcome } \\
\hline Mean satisfaction at discharge (SD) & $23.7(3.9)$ & $22.1(3.7)$ & $1.6(-5.2 \text { to } 2.0)^{a}$ & $-0.8(-4.2 \text { to } 2.6)^{a}$ & $0.39(-0.47 \text { to } 1.24)^{d}$ \\
\hline \multicolumn{6}{|l|}{ Secondary outcomes } \\
\hline $\begin{array}{l}\text { Mean attitude toward medication } \\
\text { at discharge (SD) }\end{array}$ & $3.8(3.7)$ & $2.3(4.8)$ & $1.5(-5.6 \text { to } 2.7)^{a}$ & $-1.1(-4.4 \text { to } 2.3)^{\mathrm{a}}$ & $0.31(-0.55,1.16)^{d}$ \\
\hline $\begin{array}{l}\text { Treatment continuation at } 6 \text { months } \\
\text { after discharge, } n(\%)\end{array}$ & $8(88.9)$ & $9(69.2)$ & $19.7(-12.8 \text { to } 52.1)^{b}$ & $22.1(-24.9 \text { to } 70.1)^{\mathrm{b}}$ & $0.36(0.05,2.72)^{e}$ \\
\hline \multicolumn{6}{|l|}{ Harm } \\
\hline Mean GAF score at discharge (SD) & $55.6(11.2)$ & $47.8(18.9)$ & $7.7(-23.1$ to 7.7$)$ & $4.0(-13.0 \text { to } 20.0)^{\mathrm{a}}$ & $0.44(-0.42,1.30)^{d}$ \\
\hline Average length of stay (SD), day & $66.7(40.4)$ & $66.5(17.4)$ & $1.3(-39.6$ to 37.0$)$ & $-1.7(-43.6 \text { to } 40.9)^{\mathrm{a}}$ & $0.03(-0.82,0.88)^{d}$ \\
\hline
\end{tabular}

BPRS the Brief Psychiatric Rating Scale, Cl confidence interval, GAF the Global Assessment of Functioning, SDM shared decision making

${ }^{a}$ Mean difference $(95 \% \mathrm{Cl})$ between the SDM and usual care groups

${ }^{\mathrm{b}}$ Risk difference $(95 \% \mathrm{Cl})$ between the SDM and usual care groups

${ }^{c}$ Adjusted for sex, age, illness duration, and symptom severity assessed by the BPRS at admission

${ }^{\mathrm{d}}$ Standardized mean difference $(95 \% \mathrm{Cl})$ between the SDM and usual care groups

${ }^{e}$ Risk ratio $(95 \% \mathrm{Cl})$ between the SDM and usual care groups

homogeneous quality of interventions supported by the supervision team. However, this study had several limitations. First, the sample size was too small, as we had stopped the investigation early due to slow enrollment. As a result, our trial did not have adequate statistical power to detect a difference in primary and secondary outcomes. The primary reason for the slow enrollment was the narrow eligibility criteria focusing on only those whom it was their first admission for schizophrenia. Future studies should include a longer recruitment period, conduct a multi-center trial, or use wider eligibility criteria such as including patients with history of psychiatric hospitalization. Second, duration of follow-up might be too short. A cohort study of discharged patients with schizophrenia showed that the average time to discontinuation of antipsychotics after discharge was 289 days for olanzapine and 254 days for risperidone [27]. We might need to set the duration of followup to longer than 6 months to maximize statistical power. Third, the treatment intensity of SDM was limited due to not including peers and family in the intervention. Jönsson et al. [28] considered personal supporters to be essential members of SDM in the treatment of severe mental illness; furthermore, there have been some attempts in palliative care $[29,30]$. Not providing a post-discharge program is another concern for the intensity. Fourth, there was a contamination risk because we conducted the trial in a ward where the same medical staff could be involved in both SDM and usual care groups. Thus, patients in usual care were treated by physicians who received education on the concept of SDM, although these patients never had an opportunity to receive the standardized SDM intervention. Lastly, we did not include any measure of SDM as outcome and any assessment of fidelity of the intervention; however, this limitation might not induce bias, because independent supervisors participated in regular meetings.

\section{Conclusions}

This randomized controlled trial suggests that SDM intervention is feasible during acute inpatient treatment for schizophrenia. Large trials are needed to confirm the efficacy of the SDM in patients with schizophrenia.

\section{Abbreviations}

BPRS: Brief psychiatric rating scale; CSQ-8 J: Client satisfaction questionnaire Japanese version; DAI-10: Drug attitude inventory; ICD-10: The international statistical classification of diseases, 10th revision; SDM: Shared decision making

\section{Acknowledgments}

The authors are grateful to the dedicated colleagues at Numazu Chuo Hospital for making this research possible, Dr. Ryoji Miyata for providing the DAl-10 Japanese version, and Dr. Fuminari Misawa and Dr. Sayaka Sato for giving us advice regarding the study design. The authors also would like to thank Enago (www.enago.jp) for the English language review.

\section{Funding}

This study was supported by Health and Labor Sciences Research Grant for Comprehensive Research on Disability Health and Welfare from the Japanese Ministry of Health, Labour and Welfare (H23-Seishin-Ippan-008). The funders played no role in the study design; the collection, analysis, and interpretation of data; or in the decision to submit the manuscript for publication.

\section{Availability of data and materials}

Data sharing will be considered for researchers upon request and upon institutional review board approval.

\section{Authors' contributions}

$\mathrm{Ml}$ is the principal investigator responsible for the initial draft of this manuscript, and for organizing and implementing the study. YO, the corresponding author, calculated the sample size, decided the analytic strategy and performed the statistical analysis. YO, NS, YH, and $\mathrm{HI}$ contributed to development of the SDM intervention and the study design. NS, TN, and HH contributed to the study's management. YO also revised earlier versions of the manuscript. All authors read and approved the final manuscript.

Competing interests

The authors declare that they have no competing interests. 


\section{Consent for publication}

Not applicable.

\section{Ethics approval and consent to participate}

The institutional review board of the Yokohama City University, Japan, approved this study (No.: A130321008). For reporting, we adhered to the CONSORT statement. All participants provided written informed consent or, if the participant was younger than 20 years of age, the legal guardian provided written informed consent.

\section{Author details}

'Department of Psychiatry, Yokohama City University School of Medicine, 3-9 Fukuura, Kanazawa-ku, Yokohama 236-0004, Japan. ${ }^{2}$ Research Department, Institute for Health Economics and Policy, Association for Health Economics Research and Social Insurance and Welfare, 1-5-11 Nishishimbashi, Minato-ku, Tokyo 105-0003, Japan. ${ }^{3}$ Numazu Chuo Hospital, 24-1 Nakase-cho, Numazu, Shizuoka 410-8575, Japan. ${ }^{4}$ Department of Social Psychiatry, National Center of Neurology and Psychiatry, 4-1-1 Ogawa-Higashi, Kodaira, Tokyo 187-8502, Japan.

Received: 8 August 2016 Accepted: 31 January 2017

Published online: 06 February 2017

\section{References}

1. Laine C, Davidoff F. Patient-centered medicine. A professional evolution. JAMA. 1996;275:152-6.

2. Truog RD. Patients and doctors-evolution of a relationship. N Engl J Med. 2012;366:581-5

3. Charles C, Gafni A, Whelan T. Shared decision-making in the medical encounter: what does it mean? (or it takes at least two to tango). Soc Sci Med. 1997:44:681-92.

4. Stacey D, Bennett CL, Barry MJ, Col NF, Eden KB, Holmes-Rovner M, Llewellyn-Thomas H, Lyddiatt A, Légaré F, Thomson R. Decision aids for people facing health treatment or screening decisions. Cochrane database Syst Rev. 2011;10:CD001431.

5. Hamann J, Heres S. Adapting shared decision making for individuals with severe mental illness. Psychiatr Serv. 2014:65:1483-6.

6. Deegan PE, Ph D. Shared decision making must be adopted, not Adapted. Psychiatr Serv. 2014;65:1487.

7. SAMHSA's Working Definition Of Recovery. Substance Abuse and Mental Health Service Administration, Department of Health and Human Services. 2012. http://store.samhsa.gov/product/SAMHSA-s-Working-Definition-ofRecovery/PEP12-RECDEF. Accessed 31 Jul 2016.

8. Service User Experience in Adult Mental Health: Improving the Experience of Care for People Using Adult NHS Mental Health Services. 2012. http:// www.nice.org.uk/guidance/cg136. Accessed 31 Jul 2016.

9. Shared Decision-Making in Mental Health Care. Substance Abuse and Mental Health Service Administration, Department of Health and Human Services. 2011. http://store.samhsa.gov/product/Shared-Decision-Making-inMental-Health-Care/SMA09-4371. Accessd 31 Jul 2016.

10. Hamann J, Langer B, Winkler V, Busch R, Cohen R, Leucht S. Shared decision making for in-patients with schizophrenia. Acta Psychiatr Scand. 2006;114:265-73.

11. Hamann J, Mendel R, Meier A, Asani F, Pausch E, Leucht S, Kissling W. "How to speak to your psychiatrist": shared decision-making training for inpatients with schizophrenia. Psychiatr Serv. 2011;62:1218-21.

12. Pickett SA, Diehl SM, Steigman PJ, Prater JD, Fox A, Shipley P, Grey DD, Cook JA. Consumer empowerment and self-advocacy outcomes in a randomized study of peer-led education. Community Ment Health J. 2012; 48:420-30.

13. Troquete NAC, van den Brink RHS, Beintema H, Mulder T, van Os TWDP, Schoevers RA, Wiersma D. Risk assessment and shared care planning in outpatient forensic psychiatry: cluster randomised controlled trial. $\mathrm{Br} J$ Psychiatry. 2013;202:365-71.

14. van der Krieke L, Emerencia AC, Boonstra N, Wunderink L, de Jonge $P$, Sytema S. A web-based tool to support shared decision making for people with a psychotic disorder: randomized controlled trial and process evaluation. J Med Internet Res. 2013;15:e216.

15. McCabe R, Saidi M, Priebe S. Patient-reported outcomes in schizophrenia. Br J Psychiatry. 2007;191:s21-8.

16. Ishii M, Okumura Y, Sugiyama N, Hasegawa H, Noda T, Hirayasu Y. Efficacy of shared decision making on treatment satisfaction for patients with first- admission schizophrenia: study protocol for a randomised controlled trial. BMC Psychiatry. 2014;14:1-7.

17. Overall JE, Gorham DR. The brief psychiatric rating scale. Psychol Rep. 1962; 10:799-812.

18. Miyata R, Fujii Y, Inagaki A. Reliability of the Japanese version of Brief Psychiatric Rating Scales (BPRS) [in Japanese]. Rinsho Hyoka. 1995;23:357-67.

19. Larsen DL, Attkisson CC, Hargreaves WA, Nguyen TD. Assessment of client/ patient satisfaction: development of a general scale. Eval Program Plann. 1979;2:197-207.

20. Tachimori H, Ito H. Reliability and validity of the Japanese version of Client Satisfaction Questionnaire. Seishin Igaku (Clinical Psychiatry). 1999:41:711-7.

21. Ito H, Shingai N, Yamazumi S, Sawa Y, Iwasaki S. Patient perceptions and satisfaction of psychiatric services at their discharge. Psychiatr Neurol Jpn. 1999;101:138-47.

22. Hogan TP, Awad AG, Eastwood R. A self-report scale predictive of drug compliance in schizophrenics: reliability and discriminative validity. Psychol Med. 1983;13:177-83.

23. Miyata R, Fujii Y, Inagaki A, Yagi G. Psychophardmacology for the patients with schizophrenia and their quality of life (QOL): Assessment using the Japanese version of Drug Attitude Inventory (DAl). (abstract) (in Japanese). Seishin Shinkeigaku Zasshi. 1996;98:1045-6.

24. Hall RC. Global assessment of functioning. A modified scale. Psychosomatics. 1995;36:267-75.

25. Richardson DB, Kinlaw AC, MacLehose RF, Cole SR. Standardized binomial models for risk or prevalence ratios and differences. Int J Epidemiol. 2015;44:1660-72.

26. Hamann J, Kohl S, McCabe R, Buhner M, Mendel R, Albus M, Bernd J. What can patients do to facilitate shared decision making? A qualitative study of patients with depression or schizophrenia and psychiatrists. Soc Psychiatry Psychiatr Epidemiol. 2016;51:617-25.

27. Kroken RA, Kjelby E, Wentzel-Larsen T, Mellesdal LS, Jorgensen HA, Johnsen E. Time to discontinuation of antipsychotic drugs in a schizophrenia cohort: influence of current treatment strategies. Ther Adv Psychopharmacol. 2014; 4:228-39.

28. Dahlqvist Jönsson P, Schön UK, Rosenberg D, Sandlund M, Svedberg P. Service users' experiences of participation in decision making in mental health services. J Psychiatr Ment Health Nurs. 2015;22:688-97.

29. Washington KT, Wittenberg-Lyles E, Parker Oliver D, Demiris G, Shaunfield S, Crumb E. Application of the VALUE communication principles in ACTIVE hospice team meetings. J Palliat Med. 2012;16:121004063216008.

30. Wittenberg-Lyles E, Oliver DP, Kruse RL, Demiris G, Gage LA, Wagner K. Family caregiver participation in hospice interdisciplinary team meetings: how does it affect the nature and content of communication? Health Commun. 2013;28:110-8

\section{Submit your next manuscript to BioMed Central and we will help you at every step:}

- We accept pre-submission inquiries

- Our selector tool helps you to find the most relevant journal

- We provide round the clock customer support

- Convenient online submission

- Thorough peer review

- Inclusion in PubMed and all major indexing services

- Maximum visibility for your research

Submit your manuscript at www.biomedcentral.com/submit 